\title{
Development of a multispectral imaging system for apple firmness prediction
}

\author{
Jason Sun*, Rainer Künnemeyer*, Andrew McGlone†, Philip Roweł, Sadhana Talele* \\ *School of Engineering, University of Waikato, Hamilton, New Zealand \\ $\dagger$ The New Zealand Institute for Plant and Food Research, Hamilton, New Zealand \\ $\$$ Accelerenz Limited, Hamilton, New Zealand
}

\begin{abstract}
Multispectral imaging has been studied in recent years as a means of assessing fruit firmness. Here we report on the development of a static multispectral imaging system (MSI) that was used to validate the potential of the technique for high-speed commercial grading. The system consists of a high-performance CMOS camera, four lasers, electronically controlled shutters and a location control system. It captures four spectral scattering images on the fruit. In this study 100 'Royal Gala' apples have been measured using the static MSI. The MSI measurements were applied on the intact apple and also on a flat surface of internal flesh exposed by removing a thick skin slice. The scattering profiles were fitted with a standard light diffusion model and a heuristic modified Lorentzian model. The results showed that the correlations between penetrometer firmness and model parameters were poor, with the correlation coefficient, $R$, ranging from 0.4 to 0.8 in the best circumstance of the flat surface measurements. Although multivariate models can improve the correlations, this work suggests that laser scattering information on its own is not sufficient to predict the firmness.
\end{abstract}

Keywords- Multispectral imaging; fruit; apples; firmness.

\section{INTRODUCTION}

Apple exports make up $32.4 \%$ of the total fruit exports from NZ and had a value of NZ\$536 million in 2014 [1]. Firmness is a key quality parameter. It is used to determine maturity and harvest-time, and it is used as a primary grading standard for shipping, marketing, sorting, and processing of apples. The destructive penetrometer fruit firmness tester (FF) is the industry standard measuring tool and requires the penetration of a small mechanical probe into the fruit. FF measurements are thus only suitable for sampling purposes and cannot be used for grading or sorting fruit. An optical firmness measurement technique would have many advantages as it would require no mechanical contact with the fruit, and could operate at the high speeds of a commercial fruit grader.

Light scattering is a dominant phenomenon in turbid biological materials and is influenced by fruit tissue density, cell composition, and extra- and intra-cellular structures. It is generally accepted that scattering can provide information about textural properties of fruit [2]. There have been studies measuring light scattering phenomena as a means of assessing fruit quality including firmness. Those that measure scattering images from apples at multiple wavelengths appear to be the most promising [2-11]. With this in mind we have developed a static multispectral imaging system (MSI) with the aim of validating this technique for use on commercial fruit graders.

\section{MATERIAL \& METHODS}

\section{A. Apple Samples}

Approximately 200 'Royal Gala' Apple were harvested from an orchard in Havelock North, New Zealand, and then stored in a cool room $\left(2-3{ }^{\circ} \mathrm{C}\right) .100$ apples were moved to a humidified chamber $\left(80 \%\right.$ to $85 \%$ humidity, $\left.20^{\circ} \mathrm{C}\right)$ to provide a wide distribution in firmness. Some measurements were carried out on the apples stored in the cool room in order to obtain the top range of firmness. A total of 100 apples were measured over the experimental period (50 days). The statistics of firmness for all test apples is summarized in Table I. Apple size was between 64.1 and $76.1 \mathrm{~mm}$.

\begin{tabular}{lcccc} 
TABLE I. & \multicolumn{4}{c}{ PENETROMETER FIRMNESS DSTRIBUTION OF APPLES USED. } \\
& Mean & Standard deviation & Minimum & Maximum \\
\hline Firmness $[\mathrm{N}]$ & 62.8 & 13.9 & 38.9 & 90.0 \\
\hline
\end{tabular}

\section{B. Multispectral Imaging System}

The MSI system (Figure 1) consists of four lasers at different wavelengths, each of these wavelengths was chosen for a particular purpose. The $685 \mathrm{~nm}$ laser is near the chlorophyll absorption peak, providing an indication of the chlorophyll content in fruit. The $850 \mathrm{~nm}$ laser allowed light to deeply penetrate into the apple tissue due to low absorption in this region, and the $904 \mathrm{~nm}$ laser has been shown useful for predicting firmness and soluble solids content in apples. 980 nm corresponds to a water absorption peak, which provides complementary information about the softening process.

a)

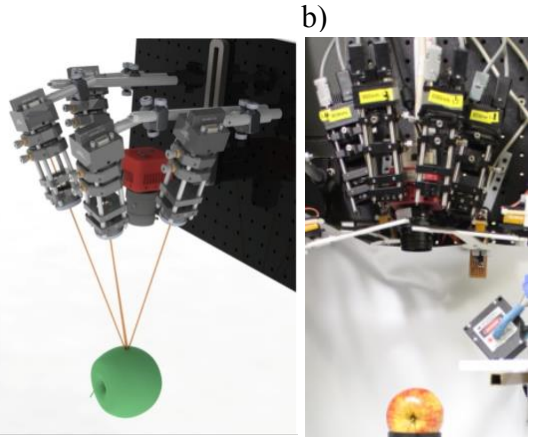

Figure 1. (a) CAD model of the static MSI system. (b) Actual implementation configured with four lasers, each with electronically controlled shutter. 
The scattering image was captured by a high-performance CMOS camera (MV1-D1312I-160-CL, Photonfocus, Switzerland). The camera was coupled with a Navitar $12.5 \mathrm{~mm}$ lens (NMV-25M1, Navitar, Rochester, New York). A fruit location control system that includes a translation stage and a laser displacement sensor was used to ensure the top surface of the fruit had a fixed distance from the camera lens. A set of electronically controlled mechanical shutters were placed in front of the lasers to enable single wavelength illumination.

The data processing technique required the laser image to be symmetric. A spatial filter was used to remove the elliptical shape of the beam and any stray light in the system. A quarter wave-plate was installed to make the laser light circularly polarised, which reduces the scattering asymmetry caused by Mie scattering.

\section{Experimental Procedure}

Measurements were carried out on both sides of an apple for each measurement cycle (Figure 2). First MSI measurements were taken on the intact surface, then GUSS fruit texture analyzer (Fruit Texture Analyser GS-20, GUSS Manufacturing Limited, South Africa) readings were recorded at the side, and finally another set of MSI measurements was taken on the cut surface.

MSI system collected 49 images at each of the four wavelengths. The apple was removed and replaced for each of the four replicates.

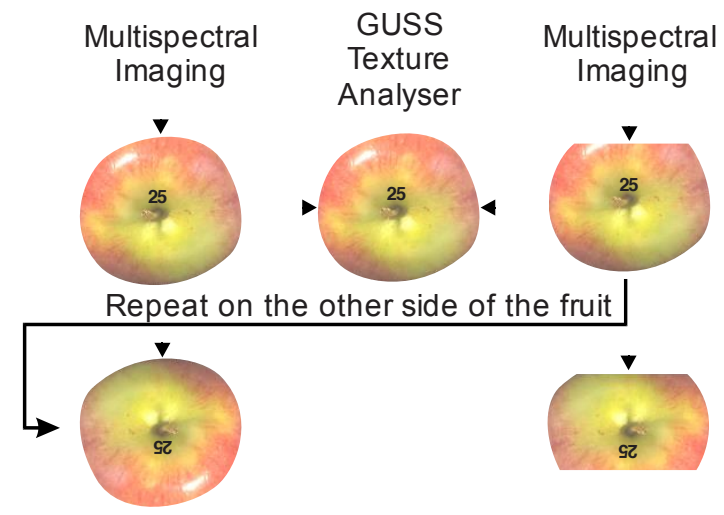

Figure 2. The sequence of the different measurements used in this study and the positions on the fruit where they occur. Measurement orientation is indicated by the position of the fruit number.

\section{Data Analysis}

A scattering image was first processed to find the centre of the illuminated area (red cross in Figure 3a). The distance to each pixel was then calculated and rounded to the nearest whole number. All pixels at each of these integer radii were grouped and averaged providing a vector of intensity values that correspond to single pixel rings expanding out from the centre point (yellow rings in Figure 3a). The intensity profile (Figure 3b) was parameterized using diffusion and Modified Lorentzian models.

The diffusion model had previously been used on 'Delicious' and 'Golden Delicious' apples [7] and was expected to be compatible with our MSI system. The modified Lorentzian function [12] is a heuristic alternative better able to handle intensity profiles from laser illumination. The model equation is:

$$
I(x)=a+\frac{b}{1+\left(\frac{|x|}{c}\right)^{d}}
$$

where $I$ is the intensity along the intensity profile, $a$ is the asymptotic value of light intensity when $x$ approaches infinity, $b$ is the peak value corresponding to the intensity at the centre of the image, $c$ is the full width half maximum (FWHM) of the intensity profile, and $d$ is related to the slope of the profile in the FWHM region. The Matlab Curve Fitting Toolbox (Mathworks, Massachusetts, United States) was used to find the best fit to the intensity profiles using the LevenbergMarquardt non-linear least squares algorithm.

For the measurements on intact apples, the intensity profile was adjusted to take account of the curved surface. The correction method [6] assumes a spherical shape of an apple and considers scattering distance and intensity signal distortion. a)

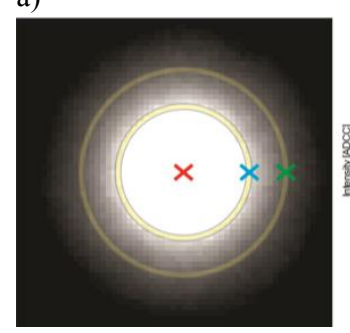

b)

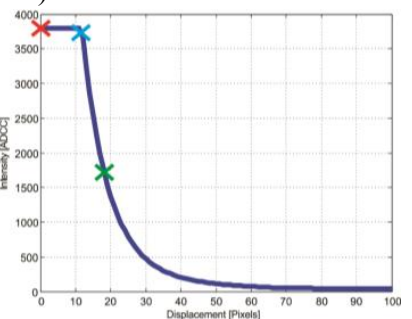

Figure 3. Illustration of radially averaging the data to reduce noise. The yellow rings indicate pixels being averaged. Each cross on the intensity profile (b) corresponds to a ring indicated on the image (a).

\section{RESULTS \& DISCUSSION}

\section{A. Reduced Scattering Coefficient}

A validation experiment was first carried out to confirm the accuracy of measuring reduced scattering coefficients at four Intralipid concentrations (Figure 4), which covers the range of typical scattering values reported for apples in the literature [13-15]. Intralipid is a fatty emulsion commonly used as a component in liquid optical phantoms, which are used to simulate light scattering and absorption properties of biological tissues.

The blue circles indicate the experimental measurements, each data point is the average of 4 replicates. The blue line is a linear fit to the data, and the red circles show the expected results based on an established empirical model [16]. At wavelength 685,850 , and $904 \mathrm{~nm}$, the scattering values are underestimated by 5,4 and $1 \%$ at $1.5 \%$ concentration respectively. At $2 \%$ concentrations, the underestimations increase to 21,20 and $16 \%$ respectively. But at 0.5 and $1 \%$ concentrations, the reduced scattering coefficients are overestimated. At $980 \mathrm{~nm}$, the MSI system overestimated at 
all four wavelengths $(21,20,16$ and $24 \%$ at $0.5,1,1.5$, and $2 \%$ concentrations).

Some studies showed similar results, which reported either under or over estimation of the reduced scattering coefficient $[13,17,18]$. The present study shows the relationship is nonetheless highly linear with an $\mathrm{R}$ of about 0.97 . Therefore this method will provide at least relative information on the behaviour of the reduced scattering coefficient in apple tissue and its possible relationship with fruit firmness.
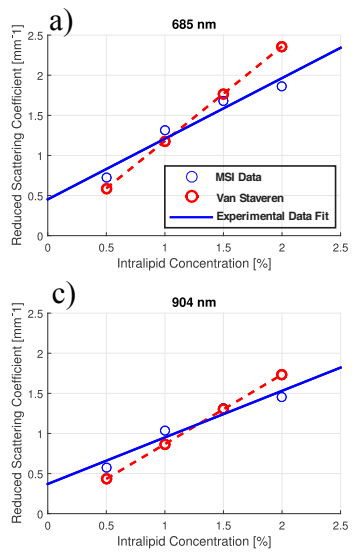
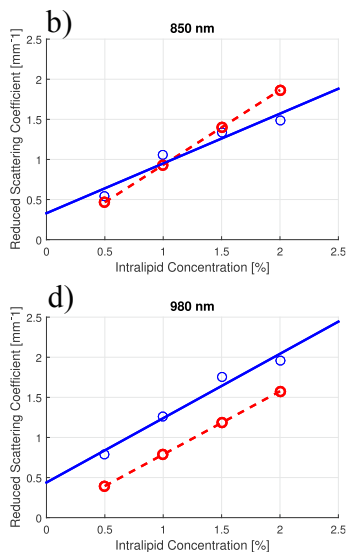

Figure 4. The reduced scattering coefficient at (a) 685, (b) 850, (c) 904, (d) $980 \mathrm{~nm}$ at four concentrations of Intralipid.

The apple measurements show the strongest correlation $(\mathrm{R}=-0.64)$ between penetrometer firmness and reduced scattering coefficient at $980 \mathrm{~nm}$ when measuring the cut surface of the apple (Figure 5a), and very poor correlation $(\mathrm{R}=-0.15)$ for intact apple measurements (Figure $5 b$ ).
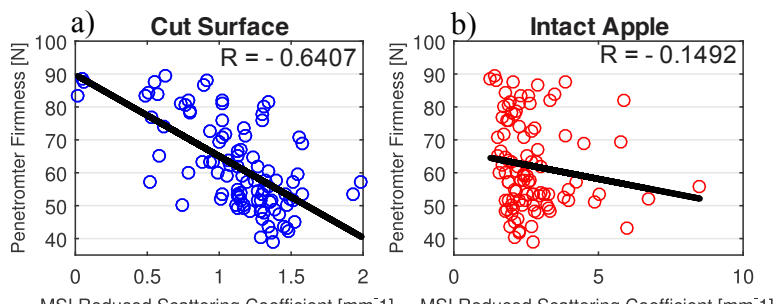

MSI Reduced Scattering Coefficient [mm-1] MSI Reduced Scattering Coefficient [mm ${ }^{-1}$ ]

Figure 5. Relationship between penetrometer firmness and the reduced scattering coefficient for measurements on (a) cut surface and (b) intact apple at $980 \mathrm{~nm}$.

For the cut surface measurements, the firmer apples had consistently lower reduced scattering coefficients compared to soft apples across all four laser wavelengths, which corresponds to negative $\mathrm{R}$ values (Table II). These results are similar to previous studies $[14,19]$.

TABLE II. CORRELATION COFFICIENT R BETWEEN PENENTROMETER FIRMNESS AND REDUCED SCATTERING COEFFICIENT AT FOUR WAVELENGTHS.

\begin{tabular}{lcccc} 
& $\mathbf{6 8 5} \mathbf{~ n m}$ & $\mathbf{8 5 0} \mathbf{n m}$ & $\mathbf{9 0 4} \mathbf{~ n m}$ & $\mathbf{9 8 0} \mathbf{n m}$ \\
\hline Cut surface & -0.61 & -0.57 & -0.64 & -0.64 \\
Intact apple & 0.50 & -0.01 & -0.15 & -0.15 \\
\hline
\end{tabular}

Intact apple measurements show a positive correlation $(\mathrm{R}=0.5)$ at $685 \mathrm{~nm}$. This does not agree with a study that found firmness decreased while reflectance at $680 \mathrm{~nm}$ increased due to a breakdown of chlorophyll [20]. The other three wavelengths gave very poor correlations.

\section{B. Modified Lorentzian Parameters}

For the cut surface measurements, parameter $a$ and $d$ provided the higher overall correlation $(\mathrm{R}=-0.63$ and -0.54 , respectively). Both parameters have the highest correlation at $980 \mathrm{~nm}$ with $\mathrm{R}=-0.78$ and 0.70 respectively (Figure 6a), which is stronger than in previous study [12].
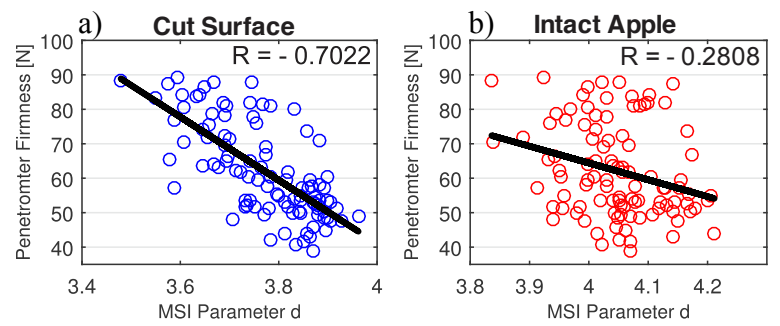

Figure 6. Relationship between penetrometer firmness and the slope (' $d$ ') parameter from the Modified Lorentzian model for measurments on (a) cut surface and (b) intact apple at $980 \mathrm{~nm}$.

However, the modified Lorentzian model showed consistently poor results for the intact apple measurements at all four wavelengths. For instance, parameter $d$ had much stronger correlation $(\mathrm{R}=-0.52)$ at $685 \mathrm{~nm}$ compared to the other wavelengths, while parameter $a$ had the highest correlation at $980 \mathrm{~nm}$ (Table III).

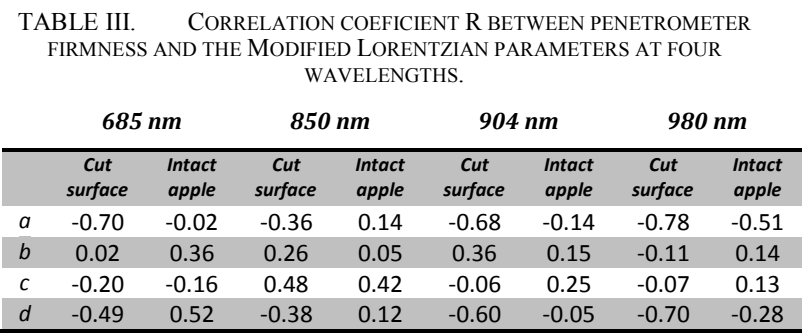

The two models have much better performance for cut surface measurements, and the results are comparable to the studies on the other cultivars of apples. Although the effects of instrument response and apple curvature were corrected using a mathematical model with the assumption of a sphere shape, the poor correlation on the intact apple suggests that this assumption may not be valid for the very inconsistently curved surface of 'Royal Gala' apples. Skin pigments can be another cause of the inferior performance on intact apples. The distinctive pigment spots can cause unusually low or high reflectance, so improvement may be achieved by removing those spots in image processing.

Our work suggests that single wavelength models are not sufficient for accurate prediction of firmness. Combinations of different parameters at different wavelengths might be useful to improve predictions. 


\section{CONCLUSION}

A multispectral imaging system with four lasers and a CMOS camera provides an efficient means of validating the technique of using laser scattering information to predict apple firmness. Comparing with previous studies, our work on "Royal Gala" achieved similar results for measurements on the cut surface, which eliminated variation caused by apple curvature and skin pigments. The correlation coefficient to the firmness ranged from -0.57 to -0.64 with reduced scattering coefficient, and -0.38 to 0.78 with the parameter $a$ and $d$ from modified Lorentzian models. The results imply that single wavelength or parameter models are not sufficient to predict firmness. The poor level of performance on the intact apple suggests that there will be significant challenges to overcome before a practical highspeed system can be developed. The challenges include skin pigment, surface curvature, high-speed data collection and processing.

\section{ACKNOWLEDGEMENT}

Jason Sun acknowledges the financial support of $\mathrm{PhD}$ scholarships from Plant and Food Research and the University of Waikato.

\section{REFERENCES}

[1] The New Zealand Institue for Plant and Food Reserach, "Fresh facts 2014," 2014.

[2] Y. K. Peng and R. F. Lu, "Analysis of spatially resolved hyperspectral scattering images for assessing apple fruit firmness and soluble solids content," Postharvest Biology and Technology, vol. 48, pp. 52-62, Apr 2008.

[3] X. Fu, Y. Ying, Y. Zhou, H. Lu, and H. Xu, "Quantitative and qualitative measurement of pear firmness based on near infrared spectroscopy and chemometrics," International Journal of Agricultural and Biological Engineering, vol. 1, pp. 69-74, 2008.

[4] X.-P. Fu, Y.-B. Ying, H.-S. Lu, H.-Y. Yu, and H.-R. Xu, "Application of some different modeling algorithms to pear MT-firmness detection using NIR spectra," Guang Pu Xue Yu Guang Pu Fen Xi/Spectroscopy and Spectral Analysis, vol. 27, pp. 911-915, 2007.

[5] R. Lu, "Nondestructive measurement of firmness and soluble solids content for apple fruit using hyperspectral scattering images," Sensing and Instrumentation for Food Quality and Safety, vol. 1, pp. 19-27, 2007.

[6] R. Lu and Y. Peng, "Development of a multispectral imaging prototype for real-time detection of apple fruit firmness," Optical Engineering, vol. 46, Dec 2007.

[7] F. Mendoza, R. Lu, D. Ariana, H. Cen, and B. Bailey, "Integrated spectral and image analysis of hyperspectral scattering data for prediction of apple fruit firmness and soluble solids content," Postharvest Biology and Technology, vol. 62, pp. 149-160, 2011.

[8] H. Min, Q. Jianwei, and L. Renfu, "Analysis of hyperspectral scattering characteristics for predicting apple fruit firmness and soluble solids content," Sensing for Agriculture and Food Quality and Safety, 73150I , 2009.

[9] Y. Peng and R. Lu, "Prediction of apple fruit firmness and soluble solids content using characteristics of multispectral scattering images," Journal of Food Engineering, vol. 82, pp. 142-152, 2007.

[10] Y. Peng and R. Lu, "A recursive method for updating apple firmness prediction models based on spectral scattering images," Optics for Natural Resources, Agriculture, and Foods II, 67610U, 2007.

[11] Y. Peng and R. Lu, "Characterization of spatially-resolved hyperspectral scattering images for assessing apple fruit firmness,"
ASAE Annual Meeting 076270, Minneapolis, MN, United states, pp. 13P-13P, 2007.

[12] Y. Peng and R. Lu, "Improving apple fruit firmness predictions by effective correction of multispectral scattering images," Postharvest Biology and Technology, vol. 41, pp. 266-274, 2006.

[13] J. Qin and R. Lu, "Determination of the optical properties of turbid materials by hyperspectral diffuse reflectance," Transactions of the ASAE: Paper No 053068, pp. 1-12, 2005.

[14] H. Cen, R. Lu, F. Mendoza, and R. M. Beaudry, "Relationship of the optical absorption and scattering properties with mechanical and structural properties of apple tissue," Postharvest Biology and Technology, vol. 85, pp. 30-38, 2013.

[15] R. Lu, M. Huang, and J. Qin, "Analysis of hyperspectral scattering characteristics for predicting apple fruit firmness and soluble solids content," Sensing for Agriculture and Food Quality and Safety, pp. 73150I-73150I-11, 2009.

[16] H. J. van Staveren, C. J. M. Moes, J. van Marie, S. A. Prahl, and M. J. C. van Gemert, "Light scattering in Intralipid-10\% in the wavelength range of 400-1100 nm," Applied Optics, vol. 30, pp. 4507-4514, 1991.

[17] Y. Zhang, X. Wen, Z. Xu, and D. Zhu, "The accuracy of a commercial spectrophotometer with single integrating sphere for measuring optical properties of turbid sample," Optical Interactions with Tissues and Cells XXI, vol. 7562, pp. 1-9, 2010.

[18] G. Zaccanti, S. D. Bianco, and F. Martelli, "Measurements of optical properties of high-density media," Applied Optics, vol. 42, pp. 40234030, 2003

[19] S. Lurie, M. Vanoli, A. Dagar, A. Weksler, F. Lovati, P. Eccher Zerbini, et al., "Chilling injury in stored nectarines and its detection by time-resolved reflectance spectroscopy," Postharvest Biology and Technology, vol. 59, pp. 211-218, 2011.

[20] L. Lleó, P. Barreiro, M. Ruiz-Altisent, and A. Herrero, "Multispectral images of peach related to firmness and maturity at harvest," Journal of Food Engineering, vol. 93, pp. 229-235, 2009. 